\title{
ESTADO DE CONOCIMIENTO DE LOS TRICHOPTERA DE CHILE
}

\section{CURRENT STATE OF KNOWLEDGE OF TRICHOPTERA OF CHILE}

\author{
Fresia Rojas A. \\ Museo Nacional de Historia Natural, Interior Parque Quinta Normal s/nº, Santiago, Chile. \\ Email: frojas@mnhn.cl
}

\begin{abstract}
RESUMEN
Se presenta una visión actualizada del conocimiento de Trichoptera de Chile; dicha fauna de insectos, caracterizada por un alto grado de endemismo, está representada actualmente por 18 familias, 32 géneros y 214 especies. Hydrobiosidae se destaca por ser la familia con mayor diversidad: 20 géneros y 47 especies y por tener el mayor porcentaje de elementos endémicos, pero Limnephilidae por su abundancia y por el gran tamaño de los representantes de sus 27 especies, resulta ser el constituyente más característico de los cursos de agua de los bosques de fagáceas subantárticos de la Patagonia. Dentro de las familias de origen gondwánico, Helicophidae es la única rica en géneros (5) y especies (15). Se destaca también que el bajo número de formas acuáticas descritas $(19,62 \%)$ no alcanza a cubrir a todas las familias representadas por los tricópteros adultos registrados en Chile.
\end{abstract}

Palabras Claves: Trichoptera, taxonomía, endemismo, investigación, Chile.

\begin{abstract}
An updated overview of the Chilean Trichoptera is given. They include a total of 18 families, 32 genera, and 214 species, and are caracterized by a high degree of endemism. The Hydrobiosidae have the highest genera (20) and species number, and percentage of endemic species. But because Limnephilidae is the more frecuent and their representatives species (27) are the greatest size, they appears the most characteristic group in subantarctic flow of water, of the fagace's forest of Patagonia. Between the gondwanic groups the only family richs in species is Helicophidae with 5 genus and 15 species. The small species number in acuatic forms described is emphasized $(19,62 \%)$.
\end{abstract}

Keywords: Trichoptera, taxonomy, endemism, research, Chile.

\section{INTRODUCCION}

El análisis que aquí se realiza representa una actualización fundamentada en la información contenida en una síntesis previa (Rojas 1995). Además de agregar la información pertinente al período transcurrido, se presenta un repaso abreviado del desarrollo histórico del conocimiento del orden Trichoptera en Chile, para precisar algunos énfasis diferentes desde mi perspectiva actual.
Las tres primeras especies consignadas para el país por Blanchard (1851) fueron: un Hydropsychidae, Hydropsyche annulicornis, actualmente en el género Smicridea; una especie de Calamoceratidae, Macronema aculeata, ahora en el género Phylloicus, y un Limnephilidae, Phryganea impluviatus, cuyo ejemplar tipo está desaparecido, pero que correspondería al género Verger. En el siglo XIX, destacan también los aportes de McLachlan (1871) y Mabille (1888). En 
el primer tercio del siglo XX, entre 1918 y 1934 , Navas nomina numerosas especies chilenas que describe escuetamente. Ellas en su mayoría serán redescritas por medio de la revisión de sus tipos, realizada por Schmid (1949). Schmid $(1952,1955 a, 1955 b, 1964,1989)$ inicia los estudios globales de las familias en el Area Neotropical, $\mathrm{y}$ hace sustanciales aportes al conocimiento de nuestras especies. Mosely (1934, 1936), Yamamoto (1966) y Jacquemar (1980a, 1980b) colaboran con contribuciones ocasionales. En la segunda mitad del siglo XX, Oliver Flint aplica al estudio de nuestras especies, una perspectiva de exploración biogeográfica de los Trichoptera en el Area Neotropical. Este autor realizó repetidas giras de recolección de material en el cono sur de América, cubriendo el territorio que llegará a distinguir como la Subregión Biogeográfica Chilena, en el que congrega a Chile desde Coquimbo al sur, junto con los lagos del sur de Argentina y toda la Patagonia. A partir de 1963, Flint ha producido una corriente de descripciones aisladas principalmente de adultos, que culmina antes de la llegada del siglo XXI, con dos importantes revisiones. La primera constituye una revisión nomenclatural (Flint et al. 1999a), precursora de la segunda, que es el Catálogo de los Tricópteros Neotropicales (Flint et al. 1999b). Flint desde los años 80 ha reorientado su trabajo y el de sus colaboradores para incluir el reconocimiento de las formas acuáticas (Flint 1981, Flint et al. 1999, Holzenthal 1986, 1988, Holzenthal \& Flint 1995). Algunas larvas y pupas del material colectado por él, han sido descritas por las entomólogas argentinas Angrisano (1997, 1998 y 2001) y Valverde (1997, 1998), abarcando especies compartidas con nuestro territorio. Sin embargo, todavía el conjunto de conocimiento logrado hasta ahora resulta incompleto. En algunas familias es posible diferenciar gran parte de las formas acuáticas de los géneros y especies (e.g. Limnephilidae, Hydrobiosidae, Hydroptilidae), pero subsiste un número importante de ellas, cuyas especies carecen de alguna descripción de los estados preimaginales. Sin embargo, la dificultad ha disminuido con la publicación de Angrisano y Korob (2001), que cubre de manera general el espectro de familias del Área Neotropical.

\section{DIVERSIDAD DE FAMILIAS Y ESPECIES}

En la Tabla I se presenta un cuadro sinóptico con la clasificación superior hasta género, y el recuento de especies del orden Trichoptera dadas para Chile. A la fecha (año 2004), las especies registradas para Chile alcanzan a 214 (columna A), lo que implica un aumento de 5,4. \% en los últimos ocho años transcurridos desde el primer recuento de 1995. En (columna B) se registra el número de las formas acuáticas conocidas para cada género $(=42)$, que resulta corresponder al 19,62\% de la diversidad de especies.

La familia más diversificada es Hydrobiosidae con 20 géneros y 47 especies reconocidas. Sin embargo, es Limnephilidae por efecto de la talla de los individuos y de su abundancia, la que resulta casi emblemática de la presencia de los tricópteros en los cursos de agua de los bosques patagónicos.

El número de familias presentes no ha variado, pero se han sustituido familias; Psychomyiidae ha desaparecido porque Astrotinodes, su único género, ha sido transferido a la familia Ecnomidae, anteriormente desconocida en esta subregión. Ecnomidae ha quedado representada por las especies de Austrotinodes, conjuntamente con el género Chilocentropus descrito originalmente en Polycentrodidae por Navas 1934. Además, la mayoría de los cambios nomenclaturales establecidos por la revisión de Flint et al. (1999a), resultaron de aplicar la ley de prioridad, al poner en conexión la línea de trabajo de Flint con las descripciones originales preexistentes. Nombres tanto específicos como genéricos de Navas han sido revalidados, así sucede con Apatanodes, Verger y Pangulia, géneros integrantes de las familias Hydrobiosidae, Limnephilidae y Kokiridae, respectivamente.

\section{DisTRIBUCIÓN GEOGRÁFICA}

La distribución geográfica conocida para el orden va de la IV a la XII Región, limitada hacia el norte por la aridez, aunque se conocen introducciones desde la Subregión Brasilera (e.g. especies de la familia Hydroptilidae en el río Loa). La mayor diversidad de especies está localizada en las regiones VIII a X, prevaleciendo la Región del Biobío con un porcentaje que supera a la mitad de todas las especies registradas para Chile. 
Tricópteros de Chile: RoJas, F.

TABLA I. Cuadro sinóptico del orden Trichoptera en Chile. A= Número de especies conocidas en Chile, B= Número de las formas acuáticas conocidas para cada género.

TABLA I. Especies de Trichoptera de Chile. $\mathrm{A}=$ number of species, $\mathrm{B}=$ number of aquatic forms known for each genus.

\begin{tabular}{|c|c|c|c|c|}
\hline Suborden & Familias & Géneros & $\mathrm{A}$ & B \\
\hline \multirow[t]{31}{*}{ SPICIPALPIA } & Hydrobiosidae & & & \\
\hline & & Amphichorema & 3 & 0 \\
\hline & & Androchorema & 1 & 0 \\
\hline & & Apatanodes & 2 & 1 \\
\hline & & Australobiosis & 2 & 0 \\
\hline & & Cailloma & 3 & 3 \\
\hline & & Clavichorema & 7 & 0 \\
\hline & & Heterochorema & 1 & 0 \\
\hline & & Iguazu & 1 & 1 \\
\hline & & Isochorema & 2 & 0 \\
\hline & & Metachorema & 2 & 0 \\
\hline & & Microchorema & 4 & 0 \\
\hline & & Neoatopsyche & 5 & 5 \\
\hline & & Neochorema & 4 & 0 \\
\hline & & Neopsilochorema & 1 & 1 \\
\hline & & Nolganema & 1 & 0 \\
\hline & & Parachorema & 1 & 0 \\
\hline & & Pomphochorema & 1 & 0 \\
\hline & & Pseudoradema & 1 & 0 \\
\hline & & Rheochorema & 4 & 4 \\
\hline & & Stenochorema & 1 & 1 \\
\hline & Glossosomatidae & & & \\
\hline & Protoptilinae & Mastigoptila & 7 & 1 \\
\hline & & Scotiotrichia & 1 & 0 \\
\hline & & Tolhuaca & 1 & 0 \\
\hline & $\begin{array}{l}\text { Hydroptilidae } \\
\text { (Hidroptilini) }\end{array}$ & Hydroptila & 1 & 0 \\
\hline & & Oxyethira & 4 & 0 \\
\hline & (Leucotrichiini) & Celaenotrichia & 1 & 1 \\
\hline & (Neotrichini) & Neotrichia & 1 & 1 \\
\hline & (Ochrotrichini) & Metrichia & 5 & 1 \\
\hline & & Nothotrichia & 2 & 0 \\
\hline $\begin{array}{l}\text { ANNULIPALPIA } \\
\text { (Superfamilias) }\end{array}$ & \multicolumn{3}{|c|}{ ANNULIPALPIA } & B \\
\hline \multicolumn{5}{|l|}{ ANNULIPALPIA } \\
\hline \multirow[t]{2}{*}{ (Philopotamoidea) } & Philopotamidae & Dolophilodes (Sortosa) & 20 & 0 \\
\hline & Stenopsychidae & Pseudostenopsichidae & 3 & 0 \\
\hline \multirow[t]{5}{*}{ (Hydropsychoidea) } & Hydropsychidae & Smicridea (Smicridea) & 14 & 0 \\
\hline & & Smicridea (Rhyacophilax) & 1 & \\
\hline & Ecnomidae & Austrotinodes & 12 & 1 \\
\hline & & Chilocentropus & 1 & 0 \\
\hline & Polycentropodidae & Polycentropus & 7 & 0 \\
\hline
\end{tabular}


Gayana 70(1), 2006

Continuación Tabla I.

\begin{tabular}{|c|c|c|c|c|}
\hline Suborden & Familias & Géneros & $\mathrm{A}$ & $\mathrm{B}$ \\
\hline \multicolumn{5}{|l|}{ INTEGRIPALPIA } \\
\hline \multirow[t]{5}{*}{ (Plenitentoria) } & Limnephilidae & Austrocosmoecus & 1 & 1 \\
\hline & & Metacosmoecus & 1 & 1 \\
\hline & & Monocosmoecus & 5 & 3 \\
\hline & & Platycosmoecus & 1 & 1 \\
\hline & & Verger & 19 & 6 \\
\hline \multirow[t]{11}{*}{ (Brevitentoria) } & Calamoceratidae & Phylloicus & 1 & 0 \\
\hline & Kokiriidae & Pangullia & 1 & 0 \\
\hline & Leptoceridae & & & \\
\hline & Triplectinae & & & \\
\hline & (Hudsonemini) & Hudsonema & 1 & 1 \\
\hline & (Triplectidini) & Triplectides & 3 & 1 \\
\hline & Leptocerinae & & & \\
\hline & (Neptosychini) & Nectopsyche & 2 & 0 \\
\hline & (Mystacidini) & Brachysetodes & 10 & 0 \\
\hline & Phylorheithridae & Mystacopsyche & 2 & 0 \\
\hline & & Psylopsiche & 3 & 0 \\
\hline \multicolumn{5}{|l|}{ (Sericostomatoidea) } \\
\hline & Anomalopsychidae & Anomalopsyche & 1 & 1 \\
\hline & & Contulma & 1 & 1 \\
\hline & Helicophidae & Alloecentrelodes & 2 & 0 \\
\hline & & Austrocentrus & 3 & 1 \\
\hline & & Eosericostoma & 2 & 1 \\
\hline & & Microthremma & 7 & 0 \\
\hline & & Pseudosericostoma & 1 & 0 \\
\hline & Helicopsychidae & Helicopsyche & 2 & 0 \\
\hline & Sericostomatidae & Chiloecia & 1 & 0 \\
\hline & & Myotrichia & 1 & 1 \\
\hline & & Notidobiella & 3 & 1 \\
\hline \multirow[t]{3}{*}{ Sericostomatoidea } & Sericostomatidae & Parasericostoma & 10 & 2 \\
\hline & Tasimiidae & Charadropsyche & 1 & 1 \\
\hline & & Trichovespula & 1 & 1 \\
\hline
\end{tabular}

\section{Endemismo en Chile}

La condición mayoritariamente endémica de las especies, sumada al hecho de que un grupo de géneros ha resultado incluido en familias exclusivas del Área Australozelandesa (Helicophidae, Tasimiidae, Kokiriidae, Philorheithridae) y Australoasiatica (Stenopsychidae), ha proporcionado el fundamento para distinguir y caracterizar a la subregión chilenopatagónica dentro del Area Neotropical. Debido a que tal distinción rebasa las delimitaciones políticas de territorio, tal endemismo no impide que compartamos especies con Argentina. Solamente Helicophidae presenta una radiación de géneros $(=5)$ y cierta riqueza de especies $(=15)$, entre las familias que se les ha asignado un origen posiblemente gondwánico.

\section{TAXA CON PROBLEMAS DE CONSERVACIÓN}

El desconocimiento de un porcentaje de formas juveniles, determina que no sea posible pronunciarse más que por una defensa generalizada para que se mantengan las condiciones viables que permitan proyectar futuras investigaciones, no obstante la creciente contaminación de los cuerpos y cursos de agua. 
Tricópteros de Chile: RoJas, F.

\section{ESPECIALISTAS EN EL GRUPO}

Aparte de los especialistas extranjeros nombrados en la introducción, mi propia línea de trabajo se ha desarrollado con intermitencias en el Museo. Esta ha sido enfocada hacia un estudio taxonómico tendiente a completar los vacíos de información, acerca de los estados preimaginales acuáticos de los tricópteros de nuestro territorio. Además la entomóloga Maritza Mercado, en la Universidad Austral, también desarrolla un trabajo centrado en el tema de todos los insectos de desarrollo acuático.

VACíOS DE CONOCIMIENTO

No obstante el gran avance representado por la publicación del catálogo de los Trichoptera Neotropicales (Flint et al. 1999), resta aún conocer un buen porcentaje de las formas juveniles acuáticas. Las descripciones de especies, casi sin excepción, se basan en estudios de genitalia de los machos adultos, principalmente colectados con las trampas de luz. El interés en el reconocimiento de las formas juveniles acuáticas ha sido una etapa secundaria al propósito primario de describir especies, y su desarrollo, de data mucho más reciente. Esto continúa siendo lento, debido a que las recolecciones de metamorfotipos resultan mucho más esporádicas y escasas, que aquellas de cápsulas vacías o con un contenido larvario, que por sí solo es muy difícil de identificar.

En ocho $(=44,4 \%)$ de las 18 familias reportadas, no existe conocimiento ni siquiera de una sola forma acuática de alguna especie emblemática de nuestro país, en parte debido al endemismo de algunas de esas familias. Por el momento, el recuento y las claves de familias generalizadas, de Angrisano y Korob (2001), que proporcionan referencias de las formas acuáticas conocidas de otras latitudes, resultan la opción más útil como herramientas para los entomólogos, que requieren información suficiente para utilizar a los tricópteros como bioindicadores.

\section{Colecciones DE REFERENCIA}

En el Museo de Historia Natural de Santiago he venido desarrollando desde 1991 una línea de investigación bibliográfica que después de 1995 quedó en un período de momentáneo receso. En estos últimos tres años ha sido reactivada, por medio de proyectos interinstitucionales que van permitiendo una recolección sistemática de material de tricópteros acuáticos, los cuales están actualmente en estado de exhaustivo procesamiento de identificación. Para los adultos, existen colecciones de referencia en nuestro Museo (MNHN), en el Museo de Concepción y en el Museo Luis Peña de la Facultad de Agronomía de la Universidad de Chile, en Santiago.

\section{AGRADECIMIENTOS}

Agradezco al Dr. O. Flint la buena disposición para proveernos de sus valiosas publicaciones, del mismo modo quiero expresar mi reconocimiento a la Dra. E. Agrisado, quien me ha prestado su ayuda para complementar los seguimientos bibliográficos.

\section{BIBLIOGRAFIA}

Angrisano, E. B. 1983. Estados preimaginales de Magellomya limnophilus Schmid 1955 y Magellomya appendiculata (Ulmer 1904) (Trichoptera: Limnephilidae). Revista de la Sociedad Entomológica Argentina 42: 325-334.

Angrisano, E. B. 1997. Contribution to the knowledge of the larvae of Hydrobiosidae. I Neopsilochorema tricarinatum and Australochorema rectispinum. En: Proceedings of the $8^{\text {th }}$ International Symposium on Trichoptera (Eds. R. Holzenthal \& O. S. Flint Jr.), pp. 15-17. Ohio Biological Survey, Columbus, Ohio.

Angrisano, E. B. 1998 Los estados inmaduros de Neoatopsyche (Trichoptera, Hydrobiosidae). Revista de la Sociedad Entomológica Argentina 57:121-125.

Angrisano, E. B. 2000. Description of the preimaginal stages of the Stenochorema crassicostum. Aquatic Insects 23 (4):289-295.

Angrisano, E. B. 2001. Las larvas de Rheochorema e Iguazu (Trichoptera: Hydrobiosidae). Revista de la Sociedad Entomológica Argentina 60 (1-4): 195-202.

Angrisano, E. B. \& P.G. Korob. 2001. Trichoptera. En: Guía para la determinación de los artrópodos bentónicos sudamericanos (rds. H.R. Fernández \& E. Domínguez), pp. 55-92. Facultad de Ciencias Naturales e Instituto Miguel Lillo, Tucumán, Argentina.

Blanchard, E. 1851. Tricópteros. En: Historia Física y Política de Chile 6 (Ed. C. Gay), pp. 135-142. Maulde \& Renou, Paris.

FLinT, O., JR. 1963. Studies of Neotropical caddis flies, I: 
Rhyacophilidae and Glossosomatidae. Proceedings of US Natural Museum 115 (3473): 453-478.

FLINT, O., JR. 1967a. Studies of Neotropical caddis flies, II: Trichoptera collected by Prof. DR. J. Illies in the Chilean subregion. Beiträge zur Neotropischen Fauna 5: 45-68.

Flint, O., JR. 1967b. Studies of Neotropical caddis flies, V: Types of the species described by Banks and Hagen. Proceedings of US Natural Museum 123 (3619): 1-37.

FLINT, O., JR. 1968. Studies of Neotropical caddis flies, VII: Trichoptera from Masatierra Isla Juan Fernández. Revista Chilena de Entomología 6: 61-64.

FuINT, O., JR. 1969. Studies of Neotropical caddis flies IX: New genera and species from the Chilean subregion (Trichoptera). Proceedings of the Entomological Society of Washington 71: 497514.

FLINT, O., JR. 1971. Life history studies on Chilean caddisflies (Trichoptera). American Philosophical Society Yearbook 1970: 312-313.

FLINT, O., JR. 1973. Studies of Neotropical caddis flies, XVI: The genus Austrotinodes (Trichoptera: Psychomyiidae). Proceedings of the Entomological Society of Washington 86: 127142 .

FLINT, O., JR. 1974a. Studies of Neotropical caddis flies, XVIII: New species of Rhyacophilidae and Glossosomastidae (Trichoptera) Smithsonian Contribution Zoology 169:1-30.

Flint, O., JR. 1974b Studies of Neotropical caddis flies, XIX: The genus Cailloma (Trichoptera: Rhyacophilidae). Proceedings of the Entomological Society of Washington 87:473484.

FLINT, O., JR. 1974c Studies of Neotropical caddis flies, XX: Checklist of the Trichoptera, or Caddisflies of Chile. Revista Chilena de Entomología 8: 8393.

Flint, O., JR. 1977. Trichoptera. En: Biota Acuática de Sudamérica Austral (Ed. S.H. Hulbert), pp. 249253. San Diego State University, California.

FLINT, O., JR. 1979. Studies of Neotropical caddis flies, XXIII: New genera from the Chilean region. Proceedings of the Entomological Society of Washington 92 (3): 640-649.

FuINT, O., JR. 1981. Studies of Neotropical caddis flies, XXVII: Anomalopsychidae a new family of Trichoptera. En: Proceedings of the $3^{\text {rd }}$ Int. Symposium of Trichoptera, pp. 75-85. Dr. W. Junk Publishers, The Hague.

FLINT, O., JR. 1982. Studies of Neotropical caddis flies, XXX: Larvae of the genera of South American Limnephilidae (Trichoptera) Smithsonian Contribution Zoology 355: 1-30.

FLINT, O., JR. 1983. Studies of Neotropical caddis flies, XXXIII: New species from austral South America (Trichoptera) Smithsonian Contribution Zoology 377:1-100.
FLINT, O., JR. 1989. Studies of Neotropical caddis flies, XXXIX: the genus Smicridea in the Chilean Subregion (Trichoptera: Hydropsychidae) Smithsonian Contribution Zoology 472:1-45.

FLINT, O., JR. 1990. Studies of the Neotropical caddis. flies, XLIII: Trichoptera collected in Chile by S. Jaquemart from 1975 a 1977. Bulletin Institut Royal Sciences Naturelles Belgique 60:115-121.

FLINT, O., JR. 1992 Studies of the Neotropical caddis. flies, XLIX: The taxonomy and de relationships of the genus Eosericostoma, with descriptions on the inmature stages (Trichoptera: Helicophidae). Proceedings of the Entomological Society of Washington 105: 494-511.

FLINT, O., JR. 1997. Studies of the Neotropical caddisflies, LIV: The Patagonian genus Austrocentrus, with the descriptions of its inmature stages (Trichoptera: Helicophidae). En: Proceedings $8^{\text {th }}$ International Symposium of Trichoptera (Eds. R.W. Holzenthal and O.S. Flint, Jr.), pp. 99-108. Ohio Biological Survey, Columbus Ohio.

Flint, O., JR. 1999. The Chilean genus Charadropsyche, with the description of its inmature stages (Trichoptera: Tasimiidae). En: Estudies of Neotropical caddis. flies LVII (Eds. H. Malicky \& P. Chantaramongkol,), pp. 99-105. Proceedings of the 9th International Symposium on Trichoptera, Chiang Mai University, Thailand.

FLINT, O., JR. 2002. Studies of Neotropical caddis flies, LX: Three new species of the Chilean genus Microthremma, with a review of the genus (Trichoptera: Helicophidae). Entomological News 113(4):225-232.

Fuint, O. \& D.G. Denning. 1989. Studies of Neotropical caddis flies, XLI: news species and records of Austrotinodes (Trychoptera: Psychomyiidae). Pan Pacific Entomology 65: 108-122.

Flint, O., JR., R.W. Holzenthal \& S.C. Harris. 1999a. Nomenclatural and systematic changes in the Neotropical caddisflies (Insecta: Trichoptera). Insecta Mundi 13:73-84.

Flint, O., JR., R.W. Holzenthal \& S.C. Harris. 1999b. Catalog of the Neotropical Caddis. flies (Insecta: Trichoptera). Columbus, Ohio. Ohio Biological Survey $56 \mathrm{pp}$.

Holzenthal, R.W. 1986. Studies in Neotropical Leptoceridae (Trichoptera), VI: Inmature stages of Hudsonema flaminii Navas and the evolution and historical biogeography of Hudsonemini (Triplectidinae). Proceedings of the Entomological Society of Washington 88:268-279.

Holzenthal, R.W. 1988. Systematics of Neotropical Triplectides (Trichoptera: Leptoceridae). Annals Entomological Society of America 81:187-208.

Holzenthal, R.W. \& O.S. Flint, JR. 1995. Studies of the Neotropical caddis. flies, LI: Systematics of the Neotropical caddis. flies genus Contulma (Trichoptera: Anomalopsychidae). Smithsonian Contribution Zoology 575:1-59.

JACQUEMART, S. 1980a. Un trichoptère Hydroptilidae 
nouveaux du nord du Chili. Metrichia thirysae sp. n. Brenesia 17: 303-318.

JACQUEMART, S. 1980b. Description de la larve et de la nymphe d'Australomyia masatierra (Schmid) et larva de d'Australomyia masafuera (Schmid) provenant d'Archipel Juan Fernández (Chili). Bulletin Institute Royale Societé Naturelle Belgique (Entomologie) 52 (26):1-11.

Mabille, P. 1891. Néuroptères. Zoologie Missions Scientifique du Cap Horn 1882-1883 6(2): 6-9.

MacLachlan, R. 1871. On news forms of the extraEuropean Trichopterous insects. Journal of the Linnean Society (Zoology) 11: 98-141.

Mosely, M.E. 1934. New exotic Hydroptilidae. Transactions of the Royal Entomological Society of London 82:137-163.

Mosely, M.E. 1936. A revision of the Triplectidinae, a subfamily of the Leptoceridae (Trichoptera). Transactions of the Royal Entomological Society of London 85:91-130.

Navas, L. 1918. Insectos chilenos. Boletín de la Sociedad Aragonesa de Ciencias Naturales 17: 212-230.

NAvAS, L. 1926. Insectos Neotrópicos $2^{\mathrm{a}}$ serie. Revista Chilena de Historia Natural 30: 326-336.

Navas, L. 1929. Algunos insectos de Chile $2^{\mathrm{a}}$ serie. Revista Chilena de Historia Natural 33:326-334.

Navas, L. 1930. Algunos insectos de Chile $4^{\mathrm{a}}$ serie. Revista Chilena de Historia Natural 34:350-366.

Navas, L. 1932a. Décadas de insectos nuevos. Década 20. Broteria (Ciencias Naturales) 1:62-85.

Navas, L. 1932b. Décadas de insectos nuevos. Década 21 Broteria (Ciencias Naturales) 1:109-119.

Navas, L. 1932c. Insectos de Argentina y Chile. $3^{\text {a }}$ serie. Revista de la Sociedad Entomológica Argentina 5: 79-86.

NAVAS, L. 1934. Insectos sudamericanos $9^{\text {a }}$ serie. Revista de la Real Academia de Ciencias Exactas, Físicas y Naturales de Madrid 31: 167-168.

RoJas, F. 1995. Trichoptera. En: Diversidad Biológica de Chile (Eds. J.A. Simonetti, M.T.K. Arroyo, A.E. Spotorno y E. Lozada), pp. 264-268. Comisión Nacional de Investigación Científica y Tecnológica, Santiago de Chile.

Schmid, F. 1949. Les trichoptères de la Collection Navas. Eos 25:305-426.

Schmid, F. 1952. Los insectos de la Isla de Juan Fernández. 4 Trichoptera. Revista Chilena de Entomología 2: 29-34.

Schmid, F. 1955a. Contribution a la connaissance des Trichopteres neotropicaux. Memories Societé Vaud Sciences Naturelles 11: 117-160.

Schmid, F. 1955b. Contribution à 1'Etude des Limnephilidae (Trichoptera) Mittelungen Schweiz Entomologie 28: 1-245.

SCHMID, F. 1964 Contribution à 1'Etude des trichoptères neotropicaux V. Tijdschr Entomologie 107: 307 339.

Schmid, F. 1989. Les Hydrobiosides (Trichoptera :Annulipalpia) Bulletin Institut Royal Sciences Naturelles Belgique 59 :1-154.

Valverde, A.C. \& L. Miserendino. 1997. Los estados inmaduros de Parasericostoma ovale (Trichoptera: Sericostomatidae). Revista de la Sociedad Entomológica Argentina 56: 33-37.

Valverde, A.C. \& L. Miserendino. 1998. Aportes al conocimiento de los estados preimaginales de Mastigoptila longicornuta (Trichoptera: Glossosomatidae). Revista de la Sociedad Entomológica Argentina 57:49-55.

Valverde, A.C. \& R. Alvariño. 1999. Descripción de los estados preimaginales de Myotrichia murina y Parasericostoma cristatum (Trichoptera: Sericostomatidae). Revista de la Sociedad Entomológica Argentina 58: 11-16.

Yамамото, T. 1966. Five news species of the caddis. fly genus Polycentropus from South America (Trichoptera: Polycentropodidae). Canadian Entomology 98: 908-912.

Fecha de recepción: 23.08.05

Fecha de aceptación: 09.12.05 